\title{
La théorie pour de rire : Sauvez la reine de Jean- Pierre Chanod
}

Jacqueline Bernard-Billiez

\section{OpenEdition}

1 Journals

Édition électronique

URL : http://journals.openedition.org/recherchestravaux/280

DOI : 10.4000/recherchestravaux.280

ISSN : 1969-6434

Éditeur

UGA Éditions/Université Grenoble Alpes

Édition imprimée

Date de publication : 30 mai 2005

Pagination : 183-195

ISBN : 0151-1874

ISSN : 0151-1874

Référence électronique

Jacqueline Bernard-Billiez, «La théorie pour de rire : Sauvez la reine de Jean-Pierre Chanod », Recherches \& Travaux [En ligne], 67 | 2005, mis en ligne le 30 septembre 2008, consulté le 08 septembre 2020. URL : http://journals.openedition.org/recherchestravaux/280 ; DOI : https://doi.org/ 10.4000/recherchestravaux.280 


\section{La théorie pour de rire: Sauvez la reine de Jean-Pierre Chanod}

L'histoire des arts et des lettres est traditionnellement segmentée par ce qu'on appelle des périodes ou des mouvements. Repères des historiens, ils sont, éventuellement, des têtes de chapitres pour les manuels. Lorsqu'il s'agit d'un passé certain, le recul donne onction et assurance au consensus culturel qui peu à peu s'est forgé, mais quand il s'agit du présent de parution, il est plus ardu d'envisager des jugements et le lecteur qui découvre ses contemporains est toujours "un Fabrice à Waterloo». Néanmoins, l'aventure de la lecturedécouverte est toujours une voie. Et, il faut admettre, bien que ce soit un îlot perdu dans l'immensité polymédiatique, que les jeunes auteurs des Éditions de Minuit sont un régal. Héritiers de l'ascèse narrative de la fin du XXe siècle, ils explorent sans limite le champ de la création romanesque. Usant du patrimoine offert par les bibliothèques du monde ou légué, plus récemment, par les linguistes et les théoriciens du texte, leurs fictions, joyeuses et graves, travaillent ad majorem gloriam Litterarum.

Ils n'ont pas d'étiquette d'école - en auront-ils un jour ? ${ }^{\text {I }}$ - mais présentent des convergences remarquables. Rupture, ironie, érudition, narration éclatée, jeux rhétoriques, forte présence énonciative de l'auteur dont le discours scintille en paillettes multinarratrices, sont quelques-uns des points qui permettent l'investigation de la littérature d'aujourd'hui. Leurs romans résonnent d'échos et déclinent des dizaines de (re)connaissances, développant avec un art consommé et minutieusement élaboré, musicothèque ou cinémathèque éclectiques. Théorie du texte, oui, mais jouissance de cette théorie plus encore.

I. Je renvoie à une intervention que j'ai faite à Rennes en mai 2000: "Le retour du narratif: le choix de l'esthétisme ludique dans les dernières années du XXe siècle» Le temps des Lettres. Quelle périodisation pour la littérature française du XXe siècle?, sous la dir. de M. Touret et F. Dugast, Presses universitaires de Rennes, 200I, p. 295-304. 
C'est ainsi que se justifie le titre de cette petite étude sur l'un de ces auteurs «sémillants ${ }^{2}$. Théorie pour de rire? Ce n'est pas si simple.

Voici donc, dans sa nudité critique, l'écriture de Jean-Pierre Chanod, un auteur grenoblois, chercheur en ingénierie linguistique dans un laboratoire de traitement automatique des langues naturelles. Deux romans, salués par la critique, sont disponibles: Sauvez la reine (1994) et Cours toujours (1997). Pas d'études encore à ma connaissance sur ces fictions, un champ vierge avec, pour seul contact, un dialogue avec les lignes qui se sont offertes. Si je ne perds pas de vue combien il est malaisé de parler d'un texte qui n'est pas partagé par tous, je vais néanmoins tenter cette gageure en déroulant les pages de Sauvez la reine. La quatrième de couverture annonce l'aventure particulière d'un personnage féminin:

Laure voulait être ethnologue, ou quelque chose comme cela, d'à la fois sauvage et distingué. Mais la forêt lui a joué un mauvais tour, la forêt profonde avec singes hurleurs, fleuves en crue, vol de jabirus. L'Amérique entière lui a joué des tours, du Mexique au Rio de la Plata. Jusqu’à cette rencontre avec un convoyeur de cargos destinés à la casse. Équarisseur d'épaves, drôle de métier. Une occasion inespérée d'en finir avec ce continent.

L'horizon d'attente que suscite cette présentation est un exotisme séduisant, mais relativement classique, sauf peut-être à être contré par le démonstratif «ce» qui désigne ce qu'il est d'usage d'appeler «Le Nouveau Monde» et qui semble ici rejeté: "en finir» mais pour revenir à quoi? C'est la question que je me suis posée, et qui a constitué un des points de départ de ma lecture. D'autre part, on trouve dans les dernières pages du roman ces mots de Laure: "La clé des songes! Vous n'êtes rien sans la clé des songes! Vous allez m’écouter. On n'a pas avancé d'un pas avec toutes ces histoires ${ }^{3}$."

Cette phrase, en forme d'avertissement a posteriori aux interlocuteurs du roman, récompense le lecteur qui se rend compte, qu'en effet, il a fait du sur place et qu'il lui serait bien difficile, sans dénaturer l'écriture, de résumer le roman. Sans cette petite phrase, la frustration de lecture serait peut-être vive. "Clé des songes", dit le texte explicitement, "clé de lecture», dit le texte implicitement. J'ajouterai que la situation de lecture est assez bien métaphorisée par un personnage un peu falot, bien que diplomate (ou justement parce qu' il est conseiller d'ambassade) Nagy, qui ne prend d'importance que pour la clausule:

Nagy sourit niaisement. [...] Il voit mal à travers le pare-brise, plisse les yeux, bouge la tête de droite et de gauche pour mieux distinguer les obstacles potentiels,

2. Ibid., p. 304 .

3. J.-P. Chanod, Sauvez la reine, Paris, Minuit, 1994, p. I77. 
trous dans la chaussée, bordures de trottoir, gravats abandonnés sans signalisation. Il a oublié d'allumer les phares. 4 (p. I89)

Ce sont ces petits signes qui m’ont invitée à lire attentivement le texte. J'ai rencontré d'une part un immense poème de la mémoire et d'autre part une réécriture allègre et parodique des "petits chéris ", comme dirait Nathalie Sarraute, du Nouveau Roman, c'est-à-dire ses options remarquables et ses repères préférés.

Le titre Sauvez la reine ne laisse pas indifférent. Il rappelle, notamment, une exclamation liée à la reine Anne d'Autriche dans Vingt ans après d'Alexandre Dumas', à la reine de cœur dans Alice au pays des merveilles mais aussi aux personnages de reines de bien des jeux vidéo d'aujourd'hui. Il est à remarquer, bien sûr, qu'il connote, mais à l'envers, l'objectif du jeu d'échecs : la sauvegarde du roi.

L'allusion au jeu d'échecs, même tangentielle, sollicite immédiatement dans l'esprit du lecteur les notions de construction, d'affrontement et de stratégie. L'attente n'est pas déçue: les avatars, les ruses du romanesque, toute l'ébullition des questions autour du récit depuis trente ans, sont là, tranquillement et narquoisement comme si Jean-Pierre Chanod reprenait à son compte les paroles de Laure, son personnage privilégié qui dit en parlant des invitées d'un raout mondain:

Regarde comme elles me jalousent. Je suis restée libre, habillée selon ma fantaisie, une échancrure ici, un liseré de cuir là, une toque sensationnelle [...]. (p. 136)

L'histoire est simple à résumer : Laure Rovigo, étudiante en anthropologie, déçue par son maître de recherche, Tim Kimson, qui lui a préféré un Indien lors d'un séjour d'étude dans la jungle, a épousé un diplomate, Nagy, qu'elle aime et qui l'ennuie. Elle demande à Geycamp, équarrisseur d'épaves et joueur d'échecs, qu'elle a connu on ne sait où - il est question d'un piquenique qui permet de déduire qu'il a pu être à l'origine de leur rencontre - de l'accompagner à une soirée à l'ambassade de France pour qu'elle ait le courage d'avouer à son mari son désir de le quitter, pour qu'il soit libre, pour qu'elle soit libre. Le couple, finalement ne se sépare pas. Il y avait un levain de drame psychotisant mais tout aussitôt balayé.

4. Ce personnage, dont le patronyme connote la langue hongroise, est peut-être astucieusement fabriqué à partir de Ignac Nagy, publiciste et écrivain hongrois (I8IO-I854) auteur, entre autres, de Élection de fonctionnaires, I842, qui ironise sur les abus de la politique de l'Ancien Régime. Parfois, visiblement, l'écrivain montre ce qu'il s'amuse à faire: Geycamp dit "qu'il a mangé du chat à Peshawar» (p. II)) ou que dans son rêve "un dromadaire bâté broute une broussaille et blatère». (p. Io)

5. Voir Vingt Ans après: "[...] D’Artagnan mit l'épée à la main et fit signe à Porthos d'en faire autant $[\ldots .$.$] Sauvez la reine! s'écria Mazarin en s'adressant au coadjuteur [...].»$ 
L'action se construit théâtralement comme une tragi-comédie classique: tout se passe en un jour, dans un lieu clos, en l'occurrence Montevideo, selon une unité garantie par ce qu'on peut appeler la quête existentielle de Laure, personnage qui doit résoudre un état de crise: quitter son mari-abri pour sauver sa personnalité. On la suit, accompagnée de son ami Geycamp, le long d'un itinéraire urbain, relativement simple, hanté, toutefois, par le célèbrissime lycanthrope : le point de départ du parcours est une chambre d'hôtel en début de soirée et le point d'arrivée est l'ambassade de France et sa réception jusqu'à l'aube. Entre ces bornes temporelles, qui sont celles même de La Dolce vita - référence qui interfere très subtilement -, le récit du trajet proprement dit, la rue, sinueuse et pleine d'imprévus, est constamment coupé de digressions, de bifurcations descriptives liées aux fantaisies de Laure dont une des raisons d'être est la mise à jour d'une épaisseur mémorielle qui reste d'une ambiguïté fort intéressante: s'agit-il d'une fiction qui prend pour objet un récit de mémoire ou d'une fiction qui transforme un éventuel référent livresque ou vécu de l'auteur? Des contradictions évidentes dans le tissu narratif invitent à ne pas prendre au pied de la lettre une histoire qui a un survêtement de vérité, comme on peut s'en rendre compte avec ce court échange dans lequel affirmation, réfutation et explication se succèdent à toute allure:

- Pardonnez-moi, j’ai complètement oublié l'heure de notre rendez-vous [...]

- Un rendez-vous?

[...] Ils n'ont jamais eu rendez-vous, cela fait des semaines qu'ils ne se sont vus. Même Geycamp qui relève ses mensonges maintenant. (p. 20)

Et Laure d'ajouter, mais nettement plus loin: «Geycamp, somme toute, est un inconnu. Un inconnu en partance.» Plus net encore, vers la fin du roman, Nagy interroge sa femme retrouvée:

- Pourquoi tu as dit à Geycamp qu'on s'est connu au Mexique? [...]

- Après tout, nous aurions très bien pu nous connaître au Mexique.

Suit alors une série de répliques dialoguées en forme de stichomythie, qui confine au délire, marquant ainsi des possibilités que l'histoire aurait pu et n'a pas employées: des ratures virtuelles en quelque sorte.

- Oui, dans les couloirs de l'hôtel Berlin à Chapultepec.

- Ou chez un guevariste repenti à Veracruz

- À Cuernavaca, dans la résidence d'Ivan Illich.

- Dont nous aurions été les disciples assidus.

- Les conseillers émérites.

- Les chauffeurs.

- Les jardiniers.

- Les chamans.

- Les dresseurs de chiens savants. 
- Nous pourrions être les assassins de Trotski.

- Les nègres d'Octavio Paz.

- Les aides de camp de Cortés

- Les enfants cachés de Cuauhtémoc. (p. I88)

Le fonctionnement textuel de Laure, l'anamnèse, constitue le générateur de l'écriture du roman. La (re)construction d'un passé qui se fabrique au présent, à la manière d'un puzzle, qui se présente comme un échafaudage essentiellement littéraire discrédite l'actualité illusoire d'une histoire exotique que, paragraphe après paragraphe, le texte enfle comme le Rio de la Plata qui bat les flancs de la ville. Laure, en quelque sorte donne une règle de ce jeu de mémoire:

Je n'ai pas de mémoire. Ou plutôt si, j’ai une mémoire à longue portée, comme un missile [...] Boum! Ma mémoire atteint des cibles lointaines, les plaies de l'enfance et de l'adolescence. Elle sème la ruine, creuse des tranchées dans les villes, des fosses brûlées, elle ébranle les tours des cathédrales. Planquez les vitraux! Patatras. Mes pensées filent. J'ai trop peur d'en perdre en route. Impossible pourtant de retenir toutes celles qui se présentent: un mouvement de foule affolée, une bousculade; à chaque seconde sa cohue. Alors j'évacue, j'ouvre grand les portes de ma mémoire, je les laisse filer pour éviter les piétinements. Mon amnésie est un geste d'urgence, un acte d'assistance à conscience en danger [... ] la mémoire est un cimetière portatif [...] ! Ne dit-on pas "gravé dans la mémoire» comme un nom sur une pierre tombale. (p. 2I)

Cette règle construit le texte. Un élément susceptible d'être un souvenir détermine un bond en avant du récit. Partons maintenant à la découverte de cette écriture mémorielle avec deux points d'appui: la mise en évidence d'un marcottage lexical qui accumule les descriptions et un système d'empilement narratif qui crée des strates narratives.

\section{Un marcottage lexical}

Ce procédé de multiplication des plantes, par lequel une tige aérienne est mise en contact avec le sol et s'y enracine, me semble une métaphore appropriée à ce qui se passe dans Sauvez la reine: un mot est lancé, agglutinant les éléments d'un paradigme dont l'extension par association de pensée constitue le texte. Comme il n'est pas question de passer l'ensemble de l'ouvrage en revue, je n'en donnerai ici que trois exemples qui m'ont semblé particulièrement remarquables.

Au début du texte, le regard indécis de Geycamp accroche un livre sur une étagère: Le Guide des égarés. Aussitôt, ce livre fait partie de l'histoire du personnage. Geycamp l'a

[...] déniché à la foire aux livres de la rue Tristan-Navaja. L'ouvrage traînait parmi des récits de voyage et des guides touristiques hors d'âge. (p. 9). 
Il a nourri les rêves de Geycamp qui ne parvient pas à se détacher de son contenu pour prendre pied dans l'éveil. Ce livre est vénérable puisqu'il s'agit d'un traité de philosophie de Maïmonide, médecin, théologien et philosophe juif du XII ${ }^{e}$ siècle, qui tenta de concilier la foi et la raison, premier intermédiaire entre Aristote et les docteurs de la Scolastique. Ce livre de la contradiction majeure souligne malicieusement le contraste entre le personnage de Geycamp, suggéré plutôt comme rustre et vieux loup de mer -

Une démangeaison de l'hypogastre précipite la fin de la sieste. Geycamp se gratte. Deux de ses doigts, l'annulaire et le majeur gauches, piétinent l'emplacement de l'irritation. (p. 8)

et le texte qu'il a trouvé par hasard:

La théologie traverserait-elle le cerveau le plus rebelle à la métaphysique comme l'huile la terre cuite d'une jarre? (p. 9)

On ne peut mieux désigner l'hébétude du lecteur qui s'engage dans le texte de Sauvez la reine où des égarements de tous ordres, thématiques et structurels, proliferent.

Un autre phénomène similaire est repérable, cette fois avec le regard de Laure. Elle attend Geycamp qui finit sa toilette et dans son champ visuel apparaît une valise

[...] vieillotte, rien de ces bagages qui font la fierté des voyageurs devant les tapis roulants des aéroports. Elle rappelle plutôt une valise dont l'achat remontait au mariage de ses parents en 1947. (p. 25)

Le texte ouvre cette «valise» pour construire une partie du passé de Laure et une partie de celui de Geycamp qui n'est fait, en réalité, que des suppositions de Laure mais qui procure une bien belle page sur les possibilités que l'auteur eût pu accorder à son personnage:

[...] l'idée germe chez Laure que la valise de Geycamp renferme un objet délictueux: faux dollars à l'effigie de Thomas Jefferson, peinture hollandaise (nature morte du XVII siècle, avec gibier d'eau, citron pelé, carafe de vin, raisins), statuette olmèque (enfant-jaguar, jade) ou dans un autre registre, repérages et projets de kidnapping conçus par le fils indigne d'un latifondiste [...] Rien de bien répréhensible, dans ces parages où se réfugient en grand nombre inventeurs, espions et notables en cavale, sans compter les poètes, tendance illuminatoire. (p. 29)

Le troisième exemple est la démonstration même de cette consigne d'écriture. Il s'agit du commentaire phonique du lieu du roman, Montevideo, superbe évocation, née de l'exploitation du signifiant:

Montevideo est un de ces noms de rêve: un nom qui serpente dans la bouche et dont les syllabes-anneaux évoquent la Conquista, le courage des premiers navigateurs, les aras, les tapirs, les Indiens roulant nus sur les dunes à l'heure où la Castille 
arme ses premières barques transatlantiques [...] Le nom de Montevideo s'enroule autour du corps, caresse le cou, de sa peau froide et lisse de serpent, puis descend le long du bras, en quête de cette main qui lui caressera la gueule, fermera ses mâchoires venimeuses. (p. 28)

et d'une minuscule réflexion de Laure, taquinant Geycamp:

Ah, vous êtes ponctuel. Quelle horreur! Ponctuel, vous vous rendez compte, ça veut dire réduit à un point, un petit point ballotté par les flots. (p. 35)

Ce qui entraîne, deux paragraphes plus loin, un très surprenant monologue intérieur de Geycamp qui fait le "point» sur sa vie de navigateur :

Hardi, marin! Un pion avance, une case se libère. Les stratégies se nouent dans le noir. Une tour se dresse où on ne l'attendait guère. Le marin fait cavalier seul. Son unique espoir est de faire $p a t^{6}$ avec la tempête. (p. 36)

Cette consigne productive entraîne un système narratif qui est de l'ordre de l'empilement, voire de ce qu'on pourrait nommer dans le domaine du multimédia, de l'hyperlien.

\section{Un empilement narratif}

Dans le Paysan de Paris, Aragon surréaliste menace malicieusement son lecteur de lui faire subir une description supplémentaire

Cette marchande de mouchoirs, ce petit sucrier que je vais vous décrire si vous n'êtes pas sages, ce sont les limites de moi-même, des vues idéales que j'ai faites de mes lois, de mes façons de penser, et je veux bien être pendu si ce passage est autre chose qu'une méthode pour m'affranchir de certaines contraintes [... $]^{7}$

J'ai repensé à cette boutade, au demeurant fort sérieuse, en lisant l'exorde du roman de Jean-Pierre Chanod, qui est une immense description du réveil d'un personnage. Mais, dès lors, j'ai été très attentive à ce phénomène d'en lisement du récit qui, depuis le Nouveau Roman, semble un fait acquis de l'écriture littéraire. C'est en général au cours de l'enlisement que surgit le commentaire métatextuel. Ce principe s'applique dès l'attaque: les quatre premières pages offrent une disposition anaphorique d'un syntagme très significatif: "Tout commence lentement.»

Fin de sieste. Tout commence lentement. La paupière gauche s'entrouvre, la droite se crispe, retient dans le noir ce qui reste à prendre de sommeil. [...] Réveil aigre-doux, mi-figue mi-raisin, œil droit, œil gauche. Des gouttelettes brunes, ceintes d'un liseré noir, se dessinent sur un fond vert amande: la couverture jaspée du Guide des égarés chauffe au soleil. Tout commence lentement. [...]

6. Position du roi, qui n'étant pas mat, est posé sur une case dont il ne peut bouger: nullité de la partie.

7. L. Aragon Le Paysan de Paris, L'Euvre poétique, t. III, Paris, LCD, 1974, p. I79. 
L'œil droit s'ouvre. La tête roule lentement sur le traversin; pivotement du cou, des épaules $[\ldots]$

Tout commence lentement. D'abord, effacer les rêves. [...] Mettre à la raison cette cohue d'ombres, les vivants et les morts qui, égaux dans la chimère, affluent à la mémoire, occupent le cerveau assoupi sans plus de vergogne qu'un campement de Cosaques: cortex, ville ouverte. [...] Reconstruire le monde par $35^{\circ}$ de latitude sud, $56^{\circ}$ de longitude ouest. Lentement, lentement. Tout ira plus vite, plus tard. Promis. (p. 7-II)

Et il faut attendre une douzaine de pages avant qu'il ne se passe, comme on dit, quelque chose qui enclenche une action: "On frappe à la porte.» (p. 19) Les pages liminaires construisent ainsi le personnage nommé Geycamp. Récupérateur d'épaves de bateaux, il s'éveille dans une chambre d'hôtel à Montevideo, lieu aussi minutieusement inventorié et topographié, avec ses dépendances utilitaires et tous les objets y afférant, que s'il s'agissait d'une publicité immobilière. On songe bien évidemment à la technique de Butor dans La Modification, lieu clos d'un wagon et lente transformation du lecteur par le personnage.

Il nous est donné de lire ce qui pourrait être au cinéma un long travelling: la récupération de la conscience chez un être entre veille et sommeil, instant d'indécision où tout se mêle, se brouille, souvenirs et actualité. Geycamp "voit», en effet, «les eaux proches du Rio de la Plata [qui] se précipitent dans le canal de Suez» (p. io). Heureux brouillage d'un instant indécidable où toutes les illusions peuvent arriver, qui a souvent fait le bonheur de la littérature fantastique. Ici, l'emploi systématique du mode généralisant, l'infinitif, rémunère cet entre-deux de la pensée. En dépersonnalisant, comme dans une recette culinaire, les énoncés, faits, gestes et sentiments de Geycamp, l'écrivain les attribue autant à lui-même, narrateur omniscient, qu'à sa créature, dans un dialogue implicite avec le lecteur: «Refermer les yeux, prolonger le rêve, palper une fois encore la terre rouge du Sinaï $[. .$.$] effacer les rêves [\ldots]$ reconstruire...» (p. Io)

Ce choix stylistique conduit avec efficacité à quelque chose de fort intéressant. C'est de son lit que Geycamp voit l'environnement de son éveil. De ce point d'origine, s'établit un jeu de focalisations visuelles: celle du personnage puis celle qui est proposée au lecteur. En effet, le personnage éveillé existe en épelant ce que sa vue accroche grâce à une série de notations lexicographiques. «Un rayon de soleil attire l'œil entrouvert vers la couverture jaspée d'un livre de bibliothèque» (p. 9) et existe alors, pour le roman en progression, le livre de Maïmonide. Puis, ce sont les boules chromées des manettes d'une baignoire absente, portant

[...] sur une pastille émaillée vissée au centre les mentions frio et caliente intégralement calligraphiées, et non les seules initiales $\mathrm{F}$ et $\mathrm{C}$; l'écriture inclinée à droite dégage, avec ses pleins et ses déliés, une saveur d'avant-guerre. (p. I4) 
Enfin, autre signe actif,

[...] les lettres $P H$, brodées sur chacune des pièces de linge y enlacent leurs boucles et leurs jambages. (p. I6)

Le lecteur s'invite à ce très lent déchiffrement du personnage: la lecture que Geycamp fait de son environnement, l'identification précise des objets et de leur signification est le code d'entrée dans le texte.

Symétriquement, chaque activité de Laure, chaque réplique qu'elle échange avec Geycamp, sont les occasions d'une description, qui stoppe sans prévenir la narration, et dont le style varie. Il y a, par exemple, le ton Guide bleu quand on entre avec Laure dans les établissements Boutros Muzafir, commerçants en tissus, rue Massini (p. 37), ton qui fait place à une voix théâtrale à déclamation cocassement dramatique, qui reproduit dans un monologue amphigourique la verve colorée qu'on attribue traditionnellement à un émigrant levantin. Il y a le ton "notice officielle de la capitainerie du port», le ton du récit classique avec l'histoire des amours malheureuses de Laure et de son maître, qui fonctionne comme un récit emboîté d'une dizaine de pages d'abord, puis s'arrête, puis reprend. (p. 80). Il y a le ton lyrique du discours utopique de Laure qui rêve de traverser l'Amérique de part en part, (p. I05), le ton ampoulé et prétentieux du consul (p. II8).

Une imperceptible liaison unit ces morceaux de texte, une parataxe qui suit le seul repère possible, le cheminement primesautier de Laure à travers la ville et ses rencontres. Mais l'effet de lecture est saisissant. Un kaléidoscope générique brouille un continuum diégétique qui ne cherche même pas à s'imposer. Renforçant cet effet de discontinuité, les questions-réponses que peuvent échanger les personnages frisent souvent l'arbitraire comme s'ils parlaient des langues étrangères. Laure et sa mémoire nous plongent dans une forêt obscure.

Mais le charme de ce texte est encore ailleurs. Il surgit d'une écriture que j'appellerais moqueuse, parodique, voire pastichielle, toujours finement ciselée.

\section{Une écriture moqueuse}

Très sérieux, ce roman joue avec la bibliothèque et crée nombre d'échos avec la littérature ou même le cinéma mais, en même temps, s'amuse de tout ce que son auteur a lu ou vu. J'ai choisi trois entrées pour montrer sa liberté de rencontre: la caricature du milieu diplomatique, le jeu citationnel, et enfin la métaphore des échecs.

\section{Une récréation: la caricature du milieu diplomatique}

Comme David Lodge s'en donnait à cour joie avec Un tout petit monde, JeanPierre Chanod épingle les hauts fonctionnaires français en place à l'étranger. 
Il caricature leurs ambitions, leurs manies et leur suffisance; l'onomastique n'est pas en reste pour connoter le conservatisme étroit: Louis Jammes est l'ambassadeur de France et Duhamel son conseiller culturel. La raison de la soirée étant un hommage rendu à la jeune peinture urugayenne, avec l'attribution d'un prix Paul-Cézanne au lauréat, on a droit à un hénaurme» malentendu qui tourne au burlesque le plus débridé. Sans dévoiler, comme lorsqu'il est question d'un roman policier, le pot aux roses, disons simplement que la plume de Chanod griffe avec ardeur autant l'ignorance que le snobisme des participants. Et c'est toujours par la parole de Laure, hantée par la nostalgie d'une vie plus sauvage ou plus naturelle, que la dénonciation advient. Elle prédit lucidement la soirée à laquelle elle a convié Geycamp:

Vous aurez de l'ambiance, une saoulerie de fonctionnaires cirrhosés par des années de coloniale, ou de post-coloniale, qu'importe. Ils s'y croient encore, glaçons, ventilateurs accrochés au plafond, moustiquaire, malaria. Même ici, où il n'y a pas un moustique [...] Ce petit monde ne pose ses regards que sur ce qui brille, tinte ou pétille. (p. 48-49)

Aucun personnage ne trouve grâce sous cette plume assassine qui trace une série de portraits désolants et désopilants à la fois :

Regardez ce soir la cour de l'ambassadeur, le bestiaire magnifique, la pestilentielle ménagerie. Regardez les lions, les singes, les crocos, le serpent à l'œil crevé, le tigre boiteux [...] (p. I20)

Mais ce n'est pas un plaisir gratuit. Ce monde zoologique et difforme dont Laure voudrait s'échapper est mortifere pour l'intelligence, la sensibilité et la création. N'est-il pas question de caveau pour désigner le bas de l'escalier, près de la porte de sortie où les rescapés de la soirée achèvent, à l'aurore, leur longue beuverie qui les met face à eux-mêmes, où la parole échangée n'a plus aucune efficacité communicatrice, elle est incohérente, bruit interne des fantasmes de chacun: «[...] la nuit ne pardonne pas, elle chasse le superflu, le gras des connaissances pédantesques, exit le gras [...]» (p. I67). Du rire de connivence culturelle on passe au sérieux d'une réflexion ontologique qui met en évidence la très faible et très fragile marge de manœuvre accordée par la société, dont la langue est strictement codée, à la pulsion individuelle. Cette lecture de l'angoisse se marque dans le jeu citationnel.

\section{Le jeu citationnel: le parcours de la reine}

Le roman de Jean-Pierre Chanod est «arrangé» sous les auspices d'Elia Kazan dont le film L'Arrangement, sorti en $1969^{8}$, est cité avec insistance. On se souvient

8. The Arrangement devait marquer le retour de la collaboration entre Kazan et Brando mais finalement c'est à Kirk Douglas qu'échoit le rôle. Adapté d'un best-seller de Kazan lui-même, 
que le héros, rebelle jusqu'à la folie, détruit son passé en brûlant la maison paternelle et peut se reconstruire enfin, grâce à l'amour d'une jeune femme libre et sincère. Sauvez la reine inverse les données: la rébellion est féminine mais contrairement au héros du film de Kazan, Laure cherche en vain dans le monde masculin un point d'équilibre qu'elle ne trouvera pas.

On saura en vérité peu de chose concernant Laure Rovigo-Nagy, sinon qu'elle a de «l'éclat» et qu'une déception amoureuse l'accable. Semblable à la reine, pièce de l'échiquier qui a la plus grande liberté de parcours, Laure est la voix dominante du roman. Ses interlocuteurs sont des faire-valoir, Geycamp le marin, Abelardo, le confident éphémère, Nagy, l'époux absent. Elle va, vient, interrompt, apostrophe donnant l'impression d'un long monologue, ce qui accentue l'aspect tragique du personnage. Elle coupe sa longue quête de citations de Dante "qu'elle aurait voulu, dit-elle tout apprendre», et particulièrement le premier chant de "L'Enfer» dans la Divine comédie, dont les premiers vers fonctionnent comme un refrain obsessionnel pour dire son impuissance à prendre une décision déchirante.

\section{Nel mezzo del cammin di nostra vita}

mi ritrovai per una selva oscura

ché la diritta via era smarrita [...] 9

Mais si l'intertextualité avec le texte de Dante est explicite, elle semble relayée par une autre intertextualité, tacite: le commentaire que Philippe Sollers fit du poème de Dante en I968 dans un article fondamental «Dante ou la traversée de l'écriture». Quelques lignes de cette étude éclairent singulièrement l'élaboration du personnage:

C'est par la parole, en effet, que l'homme occupe une position intermédiaire entre les animaux et les anges. Les premiers ont les mêmes actes et les mêmes passions à l'intérieur de la même espèce et ne communiquent pas avec les autres espèces. Les seconds sont censés communiquer directement entre eux ou encore par le moyen du miroir resplendissant du divin [...] Nous pourrions dire en somme que les uns sont dans un signifiant intégral, les autres dans un signifié pur. ${ }^{10}$

\footnotetext{
largement autobiographique, ce film est le pendant d'America, America: une réflexion sur les mythes américains de réussite sociale. Injustement méconnu, parfois classé parmi les œuvres mineures de Kazan, The Arrangement est probablement son film le plus personnel. Le réalisateur, comme à son habitude, y développe sans concession sa vision des travers du rêve américain, mais laisse à son héros une porte de sortie: il devient écrivain. Bien qu'il ne soit pas son dernier film, The Arrangement fait figure de testament. L'écriture ou la vie... Les rôles titres sont servis par Kirk Douglas dans le rôle d'Eddie et Faye Dunaway dans celui de l'accompagnatrice.

9. Au milieu du chemin de notre vie je me retrouvai par une forêt obscure car la voie droite était perdue $[\ldots]$

Io. Ph. Sollers, «Dante ou la traversée de l'écriture», Logiques, Paris, Seuil (Tel Quel), I968, p. 59.
} 
Or, Laure est souvent proche d'animaux carnassiers, physiquement et pulsionnellement, pour qui elle a une évidente sympathie: chien, louve, renarde, chat (elle a accroché des morceaux de fourrure à sa robe de soirée pour faire enrager le strict et conventionnel Nagy) et même «louve-garouve" comme elle le dit pour faire bonne mesure car nous sommes sur le territoire de Lautréamont; et Laure, anagramme partielle du célèbre patronyme se fait, ditelle, «lycanthrope urbain». "Toutes dents dehors, les lèvres crispées en gueule de coyotte, le regard sombre, le front ridé par la grimace, Laure joue au carnassier devant la glace.» (p. 42)

Mais elle est aussi liée aux anges, qu'elle redoute, en la personne de Nagy le diplomate, l'Ange par excellence, le cérébral, celui qui veut la protéger à tout prix, l'aseptiser aussi en quelque sorte, qu'elle aime et qu'elle veut fuir à la fois: "Tu parles, des anges! Des détraqués, oui.» (p. 170).

Dès lors, la figure un peu farfelue de la jeune anthropologue prend du sens. Son insatisfaction, ses doutes, ses exigences deviennent autant de symboles d'une crise d'identité. L'idée tout à fait intéressante de Chanod a été de travestir en récit de vie, dans un contexte contemporain, la quête philosophique de Dante, mais avec suffisamment de marqueurs d'irréalisme pour que Laure Rovigo - le roving, mot anglais, bien proche du patronyme fabriqué, est un enroulement de fils sans torsion - soit une figure du langage en train de chercher une hypothétique plénitude, qui se situerait dans une zone mal définie entre un passé lointain (une aventure dans la forêt avec Tim l'Anglais), un passé plus récent (une courte rencontre avec un professeur mexicain Enrique Valdecasas de la Mora qui se dit trahi par la littérature) et sa situation présente (épouse d'un diplomate raffiné et amie d'un marin bourru). Indécise, révoltée, errante, elle aime le crépuscule «cette sensation d'entre d'eux, là, maintenant, c'est parfait. Tout se mélange» (p. 90).

\section{Échec au roi: les défenses possibles}

La fiction de Jean-Pierre Chanod, mise sous le signe de l'affrontement, use de la métaphore récurrente de l'échiquier et, d'une manière moins accentuée, de celle de l'océan, pour souligner le combat inégal entre l'être humain et sa destinée. Tous les personnages, premiers rôles ou utilités, sont confrontés longuement ou brièvement à ce désordre; ils tentent de trouver des solutions individuelles de repli dans une défaite programmée.

Le plus caractérisé est l'interlocuteur privilégié de Laure, celui qui l'accompagne le plus longuement, celui dont le nom est si étrange. En effet, L'Enfer de Dante donne le début de son nom tandis que la passion des échecs, qui lui est attribuée, en donne la suite. En effet, Geryon est un démon lié aux bateaux chez Dante, qui emporte Virgile et le narrateur hors du septième 
cercle. Il appartient à une catégorie de figures dont la parole est restreinte et le personnage romanesque est, en effet, fort peu causant. "Vous comptez vos mots» (p. 95) lui reproche Laure. Par ailleurs, les joueurs d'échecs sont appelés des "camps» d'où un mot-valise: GE (r) Y(on) + CAMP, d'où l'épisode, évoqué ci-dessus, de la valise du marin qui fascine Laure. "Que contient la valise de Geycamp? (p. 28) Nous finissons donc par le savoir et le personnage peut être considéré comme un double de Laure, aussi tragique dans ses réactions car Geycamp se détourne du vivant pour se protéger. Les échecs ont pour lui une rationalité rassurante. Il pense en termes de rentabilité du coup à jouer, il ne crée que dans une matière stérile: «le pion qu'on envoie à la mort, pense-t-il, redevient sitôt qu'il perd la vie, une simple pièce de buis couchée sur la table...» (p. Io6) La langue du jeu si subtile, si précieuse soit-elle, est codée, figée, inamovible. Et Laure, qui a selon Nagy «le don de faire croître l'entropie du monde» (p. 33), qui avoue avoir «l'art de [se] placer dans des situations inextricables» (p. 74) essaie de dynamiter cette immobilité stérile et de faire jouer Geycamp sur un damier vivant jusqu'à l'entendre dire: «Une femme après tout vaut mieux qu’un roi.» (p. I07)

Mais la partie n'est pas gagnée pour autant car, en réalité c'est contre deux joueurs que bataille Laure: Geycamp et Nagy, la Bête et l'Ange, deux menaces conservatrices, l'une par son égoïsme qui l'empêche d'entrer dans une histoire autre que la sienne, l'autre par élégance culturelle et respect des préjugés:

Il sacrifie ses pions, ses fous, ses tours pour sauver la reine. C'est moi, hein dis, la reine, c'est moi la reine? [...] et la reine va où elle veut, elle dévore tout sur son passage, tu vois je connais les règles, mon bon roi Nagy, tu as hérité de la plus folle des reines. (p. 139)

Laure est-elle sortie de l'Enfer? La fin du roman ne le montre pas vraiment. Elle connaît plutôt un apaisement peut-être parce qu'elle a réussi à accomplir une partie importante de son évolution: elle a brûlé les lettres du passé, première étape d'une rédemption anthropologique et philologique. Reine noire pour Geycamp, reine blanche pour Nagy, Laure semble la culture vivante, la langue, affranchie de tous les cercles, évoluant en liberté avec humour et virtuosité, ce qui n'exclut pas pour autant le tragique comme en témoignent les dernières répliques:

- Je voudrais que tout ça se termine gaiement, dit Nagy (p. I78)

- Il faut beaucoup mentir pour aimer, dit Laure. Mentir infiniment, à la folie. On serait perdu sans le mensonge. (p. 189)

\section{Sic transit Littera}

